\title{
Efeito da Habitação sobre a Mortalidade Infantil: Evidências de um Desenho de Regressão Descontínua
}

\section{Effect of Housing on Infant Mortality: Evidence of a Regression Discontinuity Design}

Fábio Nishimura ${ }^{\mathrm{a}, \mathrm{b}}$ (iD)

\begin{abstract}
Resumo: Em 2001, o governo federal criou o Programa de Arrendamento Residencial (PAR), que contemplou 340 dos 5.570 municípios que possuem pelo menos 100.000 habitantes, com a prioridade de reduzir o déficit habitacional. Estudos teóricos na área habitacional e na área da saúde indicam que ações ligadas à construção de moradias de qualidade para a população, além de reduzirem parte do déficit habitacional, também promovem uma redução da mortalidade infantil. Porém, alguns desses estudos teóricos são carentes de um instrumental estatístico mais rigoroso e que apresentem resultados estatisticamente mais consistentes. Assim, o presente trabalho analisa o efeito do PAR sobre a mortalidade infantil, utilizando como estratégia empírica o modelo de regressão descontínua, que controla possíveis problemas de endogeneidade e garante respostas estatisticamente não viesadas. Os resultados apontam que o PAR conseguiu reduzir as mortes infantis no ano de seu início assim como nos três anos subsequentes. A fim de garantir os resultados encontrados, foram aplicados testes de robustez, que sinalizaram que o modelo está bem especificado.
\end{abstract}

Palavras-chave: Programa de Arrendamento Residencial. Mortalidade infantil. Desenho de Regressão Descontínua.

\begin{abstract}
In 2001, the Federal Government created the Residential Leasing Program (RLP), which included 340 of the 5,570 municipalities that have at least 100,000 inhabitants with the priority to reduce the housing deficit. Theoretical studies in housing and health indicate that actions related to the construction quality of housing for the population, and reduce part of the housing deficit also promote a reduction in infant mortality. However, some of these theoretical studies are lacking in a more rigorous statistical instrument and which are statistically more consistent results. The present study examined the effect of RLP on infant mortality and how empirical strategy was used the regression discontinuity design model controlling for possible endogeneity problems and ensures statistically biased answers. The results showed that the RLP can reduce child deaths in the year of its inception as well as for the subsequent three years. Still, to ensure our results, robustness tests were applied which signaled that our model is well specified.

a Universidade Federal de Rondonópolis (UFR), Faculdade de Ciências Aplicadas e Políticas (FACAP), Curso de Ciências Econômicas. Rondonópolis, Mato Grosso, Brasil.

b Universidade Federal de Mato Grosso (UFMT), Faculdade de Economia (FE), Programa de Pós-Graduação em Economia (PPGEco). Cuiabá, Mato Grosso, Brasil.
\end{abstract}


Keywords: Residential leasing program. Child mortality. Regression Discontinuity Design.

JEL Classification: I20; I28; J28.

\section{1 lntrodução}

O déficit habitacional sempre foi um problema social em grande escala para diversos países no mundo. A falta de moradia leva as pessoas a apresentarem baixos níveis de qualidade de vida, provocando a deterioração da condição humana (ONIBOKUN, 1983; UNCED, 1992; SALAU, 1990). Segundo Bouillon (2012), uma em cada três famílias na América Latina e no Caribe vive em moradias que são inadequadas para a habitação. Países como Nicarágua, Honduras, Guatemala, Peru e Bolívia possuem mais de 50\% de suas famílias morando em habitações subnormais devido ao déficit habitacional.

Como política de contenção ao déficit habitacional, alguns governos passaram a intervir no setor criando ações específicas para esse fim. Ramirez (2002) afirma que a atuação do Estado mostra diferentes níveis de intervenções, sendo no setor privado, na sociedade civil e nos beneficiados pelos programas habitacionais. Algumas dessas ações resultaram em redução do déficit habitacional, como é o caso do governo chileno. Em outros casos, como no do Equador, Guatemala, Colômbia, Honduras e Venezuela, mesmo adotando as estratégias usadas no Chile, não conseguiram reduzir seus déficits habitacionais (RUBIN, 2013).

O Brasil, preocupado com seu déficit, também elaborou e aplicou ações voltadas à política habitacional ao longo dos anos. Por volta de 1946, foi criada a instituição Fundação Casa Popular, que visava, de forma específica, erradicar as populações moradoras das favelas e de mocambos no Distrito Federal. Atualmente, existe no país o programa Minha Casa, Minha Vida, que visa essencialmente a redução do déficit habitacional dos moradores de baixa renda no Brasil, com reflexos no crescimento econômico nacional. Essas ações foram criadas com o intuito de reduzir o déficit habitacional. Porém, mesmo com tais medidas, ainda persiste em grau elevado a questão da falta de moradia e suas consequências como falta de segurança, aumento de doenças e exclusão social.

Outra medida que o governo federal criou com o intuito principal de reduzir o déficit habitacional foi o Programa de Arrendamento Residencial (PAR) por meio da Lei $\mathrm{n}^{\circ} 10.188$, de 12 de fevereiro de 2001, cuja meta principal é destinar moradias de qualidade para pessoas com baixa renda.

Além das metas de redução dos déficits de moradias, os programas e ações habitacionais trazem consigo efeitos multiplicadores na economia e que por muitas vezes não são considerados em suas metas iniciais. Diversos autores apontam que as 
reduções do déficit habitacional ocorrem em paralelo à criação de novos empregos formais e informais (HENLEY, 2005; SAKS, 2008 ZHAO; LÜ; DE ROO, 2011) e alteram a arrecadação dos impostos (TEIXEIRA; CARVALHO, 2005) e o direito de uso da terra (POLLAKOWSKI; WACHTER, 1990; PENG; WHEATON, 1994).

Acevedo-Garcia et al. (2004) propõem em sua linha de pesquisa que existe também uma relação positiva entre habitações com qualidade e a saúde das pessoas. $\mathrm{O}$ autor aponta ainda que essa relação pode ser agrupada em três categorias de análise:

a) unidades habitacionais com ligação ambiental direta com a vida das pessoas: os materiais utilizados nas habitações podem influenciar diretamente nas causas das doenças (ALLEN, 2000; DUNN, 2000);

b) habitações como uma expressão do status socioeconômico: pessoas que têm uma habitação própria são consideradas com melhor nível econômico, e essa condição está positivamente relacionada com a saúde que elas apresentam (KUH et al., 2002; ROBERT; HOUSE, 1996; WADSWORTH; MONTGOMERY; BARTLEY, 1999);

c) aspectos da localização da habitação: áreas seguras para a diversão das crianças, interação social, serviços públicos e privados (transportes e policiamento) são levados em consideração para manutenção do bem-estar e da saúde (MACINTYRE; ELLAWAY, 2000; ELLEN; MIJANOVICH; DILLMAN, 2001; ELLEN; TURNER, 1997; KAWACHI; BERKMAN, 2003; LEVENTHAL; BROOKS-GUNN, 2000).

O objetivo desta pesquisa é avaliar se o PAR, como um programa que visa destinar moradias às pessoas de baixa renda, também apresenta relação positiva com a qualidade da saúde das pessoas. Nesse sentido, o presente trabalho busca verificar se esse programa promove uma melhora na qualidade de vida das famílias, reduzindo a mortalidade infantil.

Segundo dados do The Word Factbook (2012), da Central de Inteligência Americana (CIA), em países como Nicarágua, Honduras, Guatemala, Peru e Bolívia, que foram apontados como países com alta proporção de famílias morando em habitações subnormais, a taxa de mortalidade infantil média é de $26 \%$. No México, um país com maior número de famílias morando em habitações de baixa qualidade, a taxa de mortalidade infantil chega a 16.8\%. Já a taxa de mortalidade infantil do Chile, que apresentou redução do déficit habitacional, é de $7.36 \%$, a menor taxa entre os países da América do Sul.

Acevedo-Garcia et al. (2004) reconhece, de forma geral, que um ambiente saudável e com condições dignas de moradia é requisito primordial para a redução das mortes infantis. Alguns trabalhos afirmam que esses problemas ambientais estão relacionados principalmente à pobreza dos moradores e outros enfatizam a carência gerada pelas condições físico-sanitárias (JACOB; LUDWIG; MILLER, 2013 TEKÇE; SHORTER, 1984; SENGOELGE et al., 2014; BROOKS-GUNN; DUNCAN; KLEBANOV; SEALAND, 1993; CURTIS; DOOLEY; PHIPPS, 2004). 
No entanto, será que outros fatores como programas que cuidam da saúde dos moradores, características genéticas da própria população, ou mesmo, uma característica intrínseca do município podem estar influenciando a redução da mortalidade infantil e, com isso, melhorando o bem-estar das famílias, como apresentado nos resultados dos trabalhos supracitados? O simples fato dos trabalhos não considerarem a hipótese de que os municípios são distintos em suas características, como a estrutura dos serviços de saúde, ou, ainda, de existir outros programas e ações na saúde que estão em vigor nessas localidades e, acima de tudo, haver algumas variáveis não observadas que venham a ser responsáveis pelos resultados de redução das mortes infantis pode se tornar um problema quanto à eficiência dos resultados atingidos.

Neste trabalho, a premissa inicial é de que, para avaliar políticas e programas com dados não experimentais, necessitamos de uma estratégia que construa um contra fatual que não provoque viés no resultado. Assim, conhecendo o critério de participação dos municípios no PAR, observamos que somente aqueles que possuem mais de 100.000 habitantes são elegiveis para receber o programa. Dessa forma, a estratégia utilizada neste trabalho foi o desenho de regressão descontínua, abordado inicialmente no trabalho de Thistlewaite e Campbell (1960), exatamente por haver o critério de quantidade mínima de habitantes no município, o que caracteriza uma descontinuidade e, com isso, gera robustez aos resultados evitando a endogeneidade do modelo.

Para aprofundar as discussões que norteiam esses resultados, o trabalho está dividido em mais cinco partes, além desta introdução: a segunda seção apresenta os aspectos legais, o histórico e as características fundamentais para a implantação, execução e participação dos municípios no PAR; a terceira explica a estratégia empírica utilizada neste trabalho, tratando o modelo empírico e o desenho de regressão descontínua, bem como a forma de bandwidth utilizada, a especificação polinomial e as estruturas paramétrica e não paramétrica utilizadas nas regressões; a quarta apresenta os dados utilizados, a estatística descritiva e o teste de McCrary (2008) a fim de verificar se ocorre alguma manipulação das quantidades populacionais; a quinta aborda as análises dos resultados; e a sexta traça as considerações finais.

\section{Programa de Arrendamento Residencial}

A preocupação em relação à questão habitacional brasileira sempre motivou profundas discussões entre os especialistas da área em relação aos efeitos ocorridos e às ações executadas pelos governos. Algumas propostas relacionadas às políticas habitacionais, como a Fundação da Casa Popular, em 1946, o Banco Nacional de Habitação (BNH) e o Sistema Nacional de Habitação (SNH), em 1964, o Programa de Financiamento de Lotes Urbanizados (Profi-Lurb), o Financiamento de Cons- 
trução, Conclusão ou Melhoria de Habitação de Interesse Popular (Ficam) e o Programa de Erradicação de Sub-habitação (Promorar), esses em vigor na década de 1970, foram instituídas com o propósito similar de suprir a necessidade habitacional da camada mais pobre da sociedade. Os anos da década de 1980 e de 1990 não foram expressivos em termos de políticas habitacionais, aumentando esse déficit.

Em 2001, o governo federal brasileiro, preocupado com o problema de déficit habitacional e seus possíveis desdobramentos, criou através da Lei $n^{\circ} 10.188$, de 12 de fevereiro de 2001, o PAR por meio dos órgãos da Secretaria de Desenvolvimento Urbano e do Ministério da Previdência e Assistência Social. Segundo o Ministério das Cidades (BRASIL, 2011), os objetivos do programa incluem: o fomento à oferta de unidades habitacionais e à melhoria das condições do estoque de imóveis existentes, a promoção da melhoria da qualidade de vida das famílias beneficiadas, a intervenção em áreas objeto de planos diretores, a criação de novos postos de trabalho diretos e indiretos, o aproveitamento de imóveis públicos ociosos em áreas de interesse habitacional e o atendimento aos idosos e portadores de deficiência física.

Com o passar do tempo, o PAR sofreu várias alterações e reedições para a sua adequação em relação ao cenário econômico. Também sofreu alteração quanto à responsabilidade do projeto, passando para tutela do Ministério das Cidades, mas mantendo sua execução sob domínio da Caixa Econômica Federal (Caixa). Além do Ministério das Cidades e da Caixa, há o envolvimento dos estados, do Distrito Federal e dos municípios, os quais buscam identificar os locais para implantação dos projetos, indicam as famílias a serem beneficiadas, promovem ações facilitadoras e redutoras dos custos de implantação dos projetos, tais como redução de tributos, contribuições e taxas, e aportam recursos financeiros, bens ou serviços economicamente mensuráveis necessários à realização das obras e serviços do empreendimento. Também participam as empresas da construção civil e as empresas do ramo de administração imobiliária, executando atividades técnicas e operacionais de construção e gerenciamento de contratos de arrendamento dos imóveis e dos condomínios (BRASIL, 2011).

O PAR é uma política que visa reduzir os déficits habitacionais e tirar as famílias em condições de submoradias e inseri-las em condições mínimas de bem-estar. Para isso, o programa é aplicado em municípios com mais de 100 mil habitantes, nos quais os problemas de moradia ocorrem com mais intensidade. Outro recurso que o PAR oferece é o financiamento de recursos para pessoas que possuem interesse em construir novos empreendimentos, mesmo estando em etapas diferentes da construção. Assim, imóveis ainda na planta, em fases intermediárias de construção, ou em fase de recuperação/restauração, desde que o arrendatário contemple os critérios que o torne elegível ao programa, serão beneficiados pela ação.

Como condições para acesso ao recurso, o Ministério das Cidades apresenta o seguinte processo: a proposta de aquisição e produção do empreendimento é 
apresentada à Caixa pela empresa construtora proponente; esta e o projeto do empreendimento são submetidos às análises técnica e de risco; é efetuada a análise jurídica do vendedor do imóvel, da construtora proponente, bem como da regularidade e legalidade da documentação do empreendimento; a habilitação definitiva da proposta deve respeitar o limite do orçamento do Fundo de Arrendamento Residencial (FAR) para o programa, por unidade da federação; a liberação dos recursos pelas obras executadas na construção ou recuperação é feita em parcelas mensais, creditadas na conta-corrente da empresa construtora, condicionadas ao cumprimento do cronograma físico-financeiro da obra; o poder público local identifica as famílias a serem beneficiadas; após a conclusão do empreendimento, as unidades são arrendadas às famílias que atendem aos requisitos de enquadramento no PAR; a Caixa realiza a seleção dos arrendatários por meio da análise cadastral, da apuração da renda familiar bruta e da margem de renda disponível para o comprometimento com as despesas de arrendamento.

O PAR é operado com recursos do FAR, criado exclusivamente para aplicação no primeiro, composto com recursos onerosos provenientes de empréstimo junto ao FGTS e recursos não onerosos provenientes do Fundo de Apoio Social (FAS), Fundo de Investimento Social (Finsocial), Fundo de Desenvolvimento Social (FDS), Programa de Difusão Tecnológica para Construção de Habitação de Baixo Custo (Protech) e da rentabilidade das disponibilidades do FAR (BRASIL, 2011).

O PAR adota a modalidade de arrendamento mercantil ou leasing, ou seja, segundo Brito (2009), o imóvel é parte do patrimônio do FAR e permanece como propriedade fiduciária da Caixa, que é o principal agente financiador da política pública, gestor do fundo e representante do arrendador até que as unidades habitacionais estejam quitadas. O mecanismo de leasing favorece possíveis processos judiciais em caso de inadimplência. Por fim, o arrendatário tem um período de 15 anos para decidir pela aquisição do imóvel.

\section{Estratégia Empírica}

As características não observáveis, possíveis programas ou ações executadas concomitantemente ao PAR ou, inclusive, tendências seriais são situações suficientes para que provoquem sérios problemas no modelo aqui proposto e tornem os resultados viesados. Dessa forma, tomar cuidados estatísticos mais minuciosos quanto ao instrumento econométrico a ser usado e à especificação estratégica do modelo torna-se um processo mais do que necessário.

Levando-se em consideração os problemas elencados, utiliza-se como estratégia empírica o desenho de regressão descontínua (RD), ${ }^{1}$ que tem como marco

$1 \quad$ Nosso trabalho segue a mesma estratégia de regressão descontínua adotada por Fujiwara (2015) e Smith (2014). 
teórico o trabalho de Thistlewaite e Campbell (1960). Usamos essa estratégia empírica por haver um ponto de salto de probabilidade que chamamos de ponto de corte (cutoff) e que faz parte dos critérios de adesão do município ao programa. Esse ponto de corte é exatamente em municípios com mais de 100.000 habitantes. Assim, a estratégia de identificação procura comparar os municípios que estão abaixo do ponto de corte com os municípios que estão acima dele. Os municípios de tratamento estão logo acima do cutoff e os municípios abaixo são nossos controles. Angrist e Pischke (2008) tratam da seguinte forma a descontinuidade:

$$
P\left(D_{i}=1 \mid x_{i}\right)=\left\{\begin{array}{l}
g_{1}\left(x_{i}\right) \text { se } x_{i} \geq x_{0} \\
g_{0}\left(x_{i}\right) \text { se } x_{i} \geq x_{0}
\end{array}\right.
$$

Onde: $g_{1}\left(x_{0}\right) \neq g_{0}\left(x_{0}\right)$

Podemos escrever a relação entre a probabilidade de tratamento de $x_{i}$ como:

$$
E\left[D_{i} \mid x_{i}\right]=P\left(D_{i}=1 \mid x_{i}\right)=g_{0}\left(x_{i}\right)+\left[g_{1}\left(x_{i}\right)-g_{0}\left(x_{i}\right)\right] T_{i}
$$

Onde: $T_{i}=1\left(x_{i} \geq x_{0}\right)$

Dessa forma, verificamos que a estratégia $\mathrm{RD}$ consegue identificar o efeito médio do tratamento nos indivíduos em torno do ponto da descontinuidade. Segundo Rocha e Belluzzo (2010), o pressuposto de descontinuidade formaliza a ideia de que indivíduos um pouco acima e abaixo do corte precisam ser comparáveis, exigindo que tenham uma média similar dos resultados possíveis, quando recebem e não recebem o tratamento. Assim, estimamos a seguinte equação:

$$
Y_{i}=\beta_{0}+\beta_{1} P A R+\beta_{2} T_{i}+\varepsilon_{i}
$$

na qual $Y_{i}$ é a variável de interesse do modelo. Desse modo, entende-se que PAR na equação é o programa de arrendamento residencial, que leva valor igual a um, caso o município recebe o programa, e zero, caso contrário. A variável $T_{i}$ é o valor que indica se o município está acima ou abaixo do valor de corte e, por fim, é um termo de erro.

Porém, observando-se os dados, alguns municípios com o passar dos anos variam sua população, ou seja, em alguns anos estão acima do corte e em outros anos estão abaixo do corte, o que gera correlação entre o termo de erro e a variável de interesse. Dessa forma, foi escolhido o modelo de regressão descontínua fuzzy (FRD), o qual, segundo Trochim (1984), tem a sensibilidade de considerar um aumento de probabilidade, mas não de zero para um, pois a atribuição ao tratamento pode depender de fatores adicionais. Assim, para estimar os efeitos do PAR em um modelo FRD, usamos a abordagem de variáveis instrumentais (IV) proposta por Angrist e Pischke (2008), através do modelo de mínimos quadrados em dois estágios (2SLS). Dessa forma, temos: 


$$
\begin{aligned}
& Y_{i p}=\beta_{0}+\beta_{1} P A R+f\left(\text { Pop }_{i p}, \text { Cut }_{i p}\right)+X_{i p} \Theta+\varepsilon_{1 i p} \\
& P A R_{i p}=\delta_{0}+\delta_{1} \text { Cut }_{i p}+f\left(\text { Pop }_{i p}, \text { Cut }_{i p}\right)+X_{i p} \Omega+\varepsilon_{2 i p}
\end{aligned}
$$

em que Cut $t_{i p}$ é uma variável dummy que possui valor igual a um se o município tem população acima do ponto de corte. A função $f\left(P_{\text {Opp }}\right.$; Cut $\left._{i p}\right)$ é um polinômio de segunda ordem que interage com $\mathrm{Cut}_{i p} . X_{i p}$, que é um de vetor covariáveis ${ }^{2}$ com características municipais, das equipes e serviços na saúde e das condições socioeconômicas e habitacionais.

Consideramos também na estimação o modelo em sua forma paramétrica e não paramétrica. Determinamos que a função kernel seja triangular, e os bandwidths ótimos seriam determinados através da mesma estratégia adotada por Smith (2016), na qual foram usados os bandwidths ótimos de Calonico, Cattaneo e Titiunik (2014), (CCT), de Imbens e Kalyanaraman (2012), (IK), e o método Cross-Validation (CV) de Ludwing e Miller (2007) com o objetivo de determinar quanto seria a população que ficaria abaixo e acima do ponto de corte. O modelo será calculado em sua forma linear e quadrática. ${ }^{3}$

Para testar a especificação do modelo, foram aplicados quatro testes de robustez. O primeiro teste serviu para verificar possíveis tendências anteriores ao início do $\mathrm{PAR}$, assim os resultados das regressões, do ano imediatamente anterior à entrada do PAR, poderiam ou não apresentar significância estatística. Isso revelou se outros programas, ações ou tendências temporais existentes nos municípios tratados antes do PAR influenciaram ou não os resultados calculados, isolando os efeitos do PAR.

Para o segundo teste, realizamos uma alteração no cutoff, na qual arbitrariamente se alteramos o ponto de corte para 90.000 e 110.000 habitantes, com bandwidth de 10.000, que nos permitiu trabalhar com duas regressões distintas, primeiro somente com quem não é considerado tratado e depois somente com quem são considerados tratados. Assim, para ambas as regressões, não podem existir resultados com significância estatística. Isso revelou que o PAR, que apresenta um cutoff de 100.000 , realmente possui, ou não, um ponto de corte nos dados que produzem os efeitos esperados, portanto, verificar que nenhum outro ponto próximo ao cutoff poderia também ser responsável pelo resultado estimado, o que garante que as mortes infantis são reduzidas pelo PAR.

O terceiro teste de robustez utilizou variáveis dependentes não relacionadas com o PAR, que, nesse caso, são as mortes por diabetes, neoplasias e abortos. Esse teste revelou se os resultados foram conforme se esperava, ou seja, se apresenta-

2 Incluem-se covariáveis no modelo, pois, segundo Imbens e Lemieux (2008), inserir covariáveis aumenta a sua precisão.

3 A especificação do polinômio do modelo segue a orientação dada no artigo de Gelman e Imbens (2014). 
ram significância, validando o nosso modelo, e se demonstraram se o programa tem resultados eficientes, se não é apenas uma coincidência estatística.

Como último teste, verificamos a robustez do modelo através da mudança da função de kernel, utilizando-se as especificações Epanechnikov e Uniforme, sendo que todos os resultados devem apresentar significância estatística. Esse teste revela que, mesmo mudando-se a função de distribuição da regressão, os resultados são robustos.

Todos os testes fortalecem os resultados do modelo especificado, explicando a relação e os efeitos ocasionados pelo PAR sobre as mortes infantis. Na próxima seção, apresentaremos os dados utilizados para avaliar os efeitos do PAR sobre a mortalidade infantil.

\section{Dados}

A variável dependente "mortes infantis" foi coletada do Departamento de Informática do Sistema Único de Saúde (DATASUS, [2013]), órgão do Ministério da Saúde, no período de 2000 a 2004, para todos os municípios brasileiros, onde foram levantados e utilizados em seus valores per capita.

Os dados utilizados nesta pesquisa referentes ao PAR foram extraídos do banco de dados da Caixa Econômica Federal (CAIXA, 2014), que é o órgão responsável pelo gerenciamento operacional do programa, e, desse conjunto de dados, observamos que, em 2001, o programa contemplou 340 dos 5.570 municípios brasileiros existentes. Para os municípios que receberam os benefícios do PAR, denominamos de tratados (1) e de controles (0), caso contrário.

Entretanto, antes de aplicarmos a estratégia empírica deste estudo e chegarmos aos resultados, analisamos o critério imposto pelo programa, o qual orienta que os municípios contemplados devem ter mais de 100.000 habitantes. Essa imposição numérica gera margem para discussão sobre a questão da contagem da população dos municípios, o que também foi levantado no trabalho de Monasterio (2013). Gestores municipais interessados em receber o PAR podem, de certa forma, tentar manipular as informações populacionais para serem contemplados com o programa. Para testar essa hipótese, utilizamos a estratégia apresentada em McCrary (2008) e verificamos, através do histograma (ver Figura 1), que a densidade das cidades de menor população, ou seja, que estão abaixo de 100.000 habitantes, é maior que a densidade das cidades com maior população, que possuem mais de 100.000 habitantes. Assim, verificamos que os gestores municipais das cidades não manipularam o número de sua população com o intuito de receber o benefício do PAR. 
Figura 1 - Histograma da população

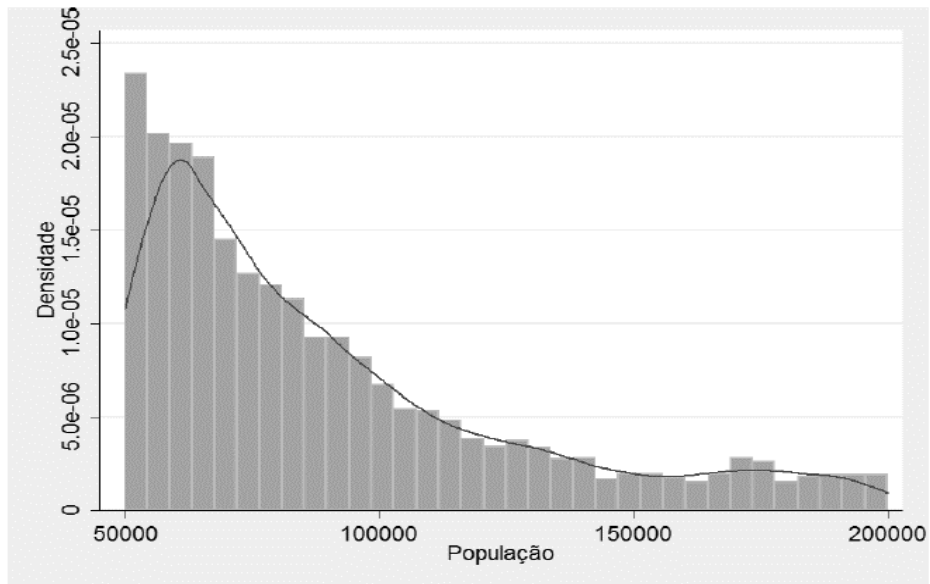

Fonte: Elaboração própria.

As covariáveis ou variáveis de controle foram obtidas de diversas fontes: do Instituto Brasileiro de Geografia e Estatística (IBGE), do Departamento de Informática do Ministério da Saúde (DATASUS, [2013]) e do Programa das Nações Unidas para o Desenvolvimento (PNUD, [2013]). Elas estão relacionadas com a infraestrutura de saúde existente, equipes e pessoal ocupado na saúde, ações epidemiológicas e serviços na saúde, características socioeconômicas, geográficas, sanitárias e índice de desenvolvimento humano municipal. Todas essas covariáveis estão a nível municipal e presentes para o período de 2000 a 2004.

\section{Análise dos Resultados}

Inicialmente, analisamos a Figura 2 com o intuito de motivar nossa estratégia empírica. Essa figura avalia a relação entre a densidade populacional dos municípios e sua relação com o PAR. Observamos que existe uma descontinuidade em relação a municípios com mais de 100.000 habitantes quando comparados a municípios com menos de 100.000 habitantes, sendo que a diferença de densidade populacional entre esses dois grupos separados pela descontinuidade chega a 0,714 - conforme os resultados da Tabela 3, apresentada mais adiante neste artigo. 
Figura 2 - População sobre o PAR

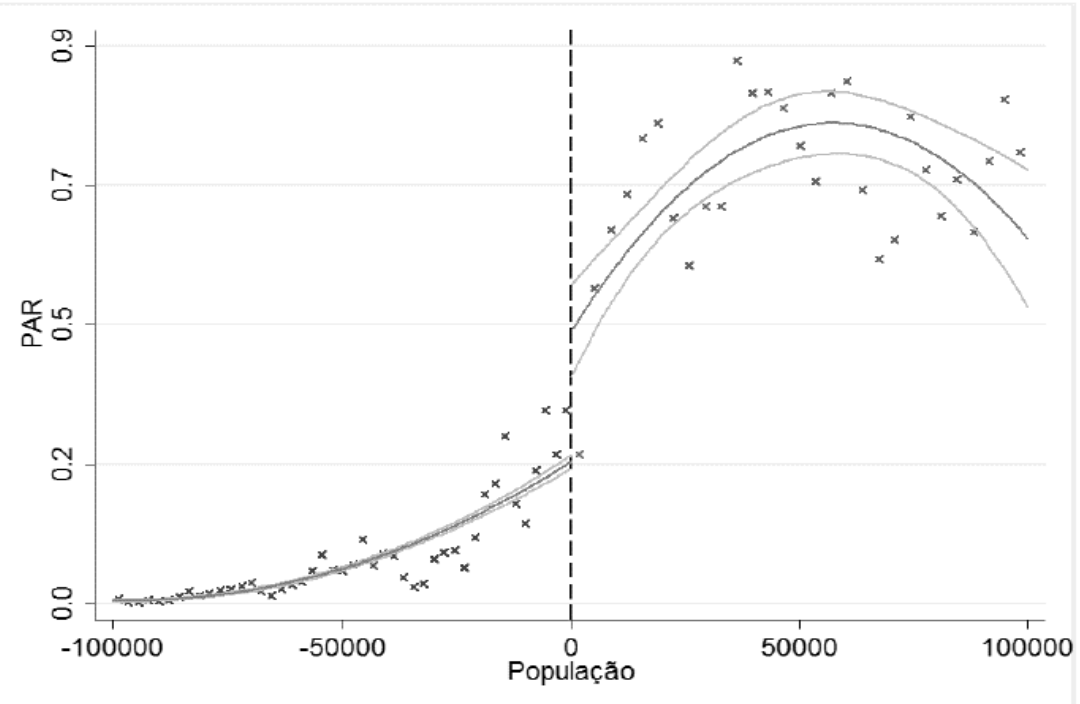

Fonte: Elaboração própria.

Isso demonstra que o critério de seleção para adoção do programa PAR nos municípios existe exatamente nas condições pré-estabelecidas da política.

Tabela 1 - Estimativa RD: População e PAR

\begin{tabular}{llllll}
\hline Variável (1) (2) & (3) & (4) & (5) & (6) &
\end{tabular}

Painel A: sem covariáveis

$\begin{array}{lcccccc}\text { Paramétrico } & 0,634^{* * *} & 0,642^{* * *} & 0,641^{* * *} & 0,641^{* * *} & 0,675^{* * *} & 0,714^{* * *} \\ \text { PAR } & (0,028) & (0,023) & (0,027) & (0,024) & (0,019) & (0,018) \\ & & & & & & \\ \text { Não Paramétrico } & 0,615^{* * *} & 0,641^{* * *} & 0,625 * * * & 0,639 * * * & 0,669 * * * & 0,708^{* * *} \\ \text { PAR } & (0,029) & (0,024) & (0,029) & (0,024) & (0,020) & (0,018)\end{array}$

Painel B: com covariáveis

continua...

Análise Econômica, Porto Alegre, v. 38, n. 76, p. 171-196, jun. 2020. 
conclusão.

\begin{tabular}{lcccccc}
\hline Variável & (1) & (2) & (3) & (4) & (5) & (6) \\
\hline Painel B: com covariáveis & & & & & \\
Paramétrico & $0,521^{* * *}$ & $0,565^{* * *}$ & $0,528^{* * *}$ & $0,564^{* * *}$ & $0,629^{* * *}$ & $0,666^{* * *}$ \\
PAR & $(0,032)$ & $(0,023)$ & $(0,031)$ & $(0,024)$ & $(0,019)$ & $(0,018)$ \\
& & & & & & \\
Não Paramétrico & $0,503^{* * *}$ & $0,564^{* * *}$ & $0,513^{* * *}$ & $0,561^{* * *}$ & $0,622^{* * *}$ & $0,659^{* * *}$ \\
PAR & $(0,033)$ & $(0,024)$ & $(0,032)$ & $(0,024)$ & $(0,020)$ & $(0,018)$ \\
& & & & & & \\
Especificação & Linear & Linear & Linear & Quadrático & Quadrático & Quadrático \\
Bandwidth & CCT & IK & CV & CCT & IK & CV \\
N. Obs. & 863 & 2.151 & 893 & 2.129 & 7.474 & 13.554 \\
\hline
\end{tabular}

Fonte: Elaboração própria.

Nota: Var. Dependente População Municipal. Todas especificações usam Kernel Triangular. O PAR estima a descontinuidade de municípios logo acima de 100.000 habitantes. CCT refere-se a seleção de bandwidth de Calonico, Cattaneo e Titiunik (2014); IK é de Imbens e Kalyanaraman (2012); CV é o método Cross-validation de Ludwig e Miller (2007). Erro-Padrão robusto em parênteses. ${ }^{* * *} \mathrm{p}<0,01 ;{ }^{* *} \mathrm{p}<0,05 ;{ }^{*} \mathrm{p}>0,1$.

O grande problema é que o município dificilmente mantém sua população constante durante os anos, logo entendemos que uma simples comparação populacional frente à ocorrência do programa não consegue avaliar de forma consistente o impacto que advém de suas ações. Dessa forma, nos tópicos seguintes apresentamos os resultados da aplicação do modelo de regressão descontínua fuzzy para identificar o efeito do PAR sobre a mortalidade infantil. Os resultados foram calculados conforme descrito na estratégia empírica deste trabalho, por meio de modelos paramétricos e não paramétricos, utilizando-se bandwidth ótimo - CCT, IK e CV - com especificação linear e quadrática e também com o uso de variáveis de controle a nível municipal. Por fim, são aplicados testes de robustez a fim de averiguamos se o modelo está bem especificado.

\subsection{Impacto do Programa de Arrendamento Residencial sobre a Mortalidade Infantil}

O teste inicial para se verificar o efeito do PAR sobre a mortalidade infantil é apresentado na Figura 3. Observamos que, exatamente no ponto de corte, ocorre uma descontinuidade, revelando que os municípios que estão após o ponto de corte apresentam uma redução nas mortes infantis em relação aos municípios que 
estão imediatamente antes do corte. O interessante dessa análise é que, devido à metodologia utilizada, as características existentes nos municípios que estão ao entorno do corte são as mesmas. Assim, a única diferença é o PAR, deixando evidente que consegue criar condições para a redução das mortes infantis.

Figura 3 - Efeito sobre as mortes infantis

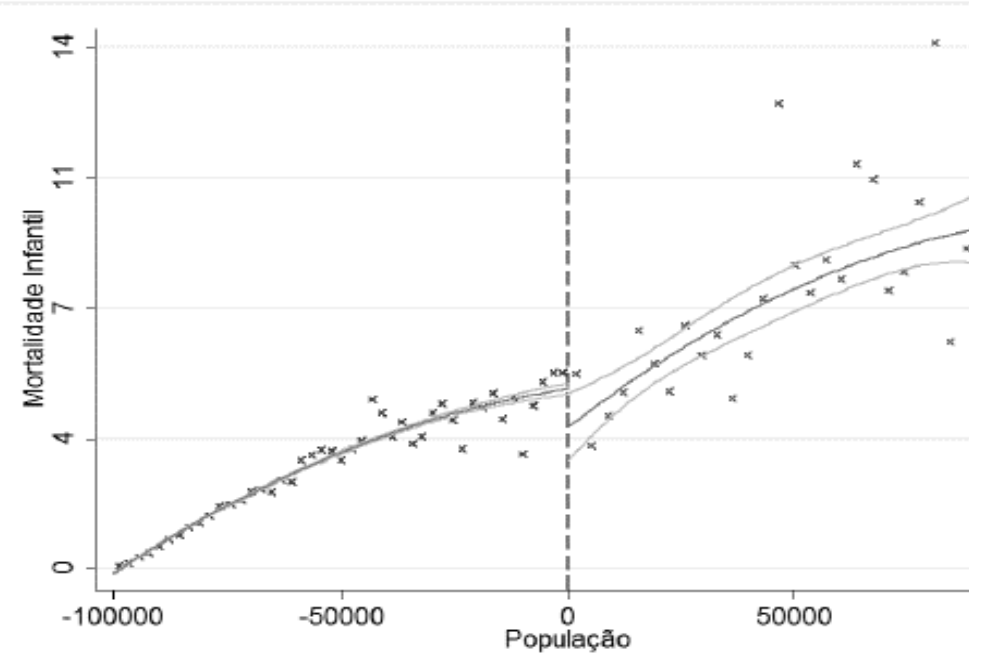

Fonte: Elaboração própria.

A Tabela 2 vem a confirmar o impacto que foi identificado na Figura 3, de que o programa proporciona a redução das mortes infantis. Esses resultados apontam que, nos municípios com mais de 100.000 habitantes e que participam do PAR, as mortes infantis têm uma redução média de 13,7\%, ou seja, o PAR, através de seus efeitos multiplicadores na economia e na sociedade, consegue reduzir as mortes das crianças menores de um ano de vida. Esses resultados são todos significantes estatisticamente e podem ser observados em todas as colunas da Tabela 2 .

Tabela 2 - RD Estimativas do Impacto do PAR sobre a Mortalidade Infantil - Sem Variáveis de Controle

\begin{tabular}{llllllll}
\hline Variável & (1) & (2) & (3) & (4) & (5) & (6) & (7) \\
\hline
\end{tabular}

Painel A: Efeito do PAR

Paramétrico

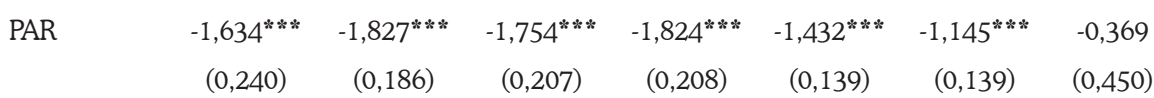

continua...

Análise Econômica, Porto Alegre, v. 38, n. 76, p. 171-196, jun. 2020. 
conclusão.

\begin{tabular}{|c|c|c|c|c|c|c|c|}
\hline Variável & (1) & (2) & (3) & (4) & (5) & (6) & (7) \\
\hline \multicolumn{8}{|c|}{ Não Paramétrico } \\
\hline PAR & $\begin{array}{c}-1,637^{* * *} \\
(0,240)\end{array}$ & $\begin{array}{c}-1,875^{* * *} \\
(0,186)\end{array}$ & $\begin{array}{c}-1,811^{* * *} \\
(0,206)\end{array}$ & $\begin{array}{c}-1,808^{* * *} \\
(0,207)\end{array}$ & $\begin{array}{c}-1,429^{* * *} \\
(0,138)\end{array}$ & $\begin{array}{c}-1,437^{* * *} \\
(0,138)\end{array}$ & $\begin{array}{l}-0,391 \\
(0,294)\end{array}$ \\
\hline
\end{tabular}

Painel B: Efeito do PAR - Anos Posteriores

Paramétrico

$\begin{array}{cccccc}-1,208^{* *} & -1,125^{*} & -1,290^{* * *} & -1,302^{* * *} & -0,973^{* * *} & -0,926^{* * *} \\ (0,474) & (0,295) & (0,414) & (0,410) & (0,314) & (0,313) \\ -1,210^{* *} & -1,117^{* * *} & -1,122^{* * *} & -1,151^{* * *} & -0,955^{* * *} & -1,028^{* * *} \\ (0,493) & (0,385) & (0,447) & (0,444) & (0,335) & (0,333) \\ -2,427^{* * *} & -2,311^{* * *} & -2,323^{* * *} & -2,391^{* * *} & -1,905^{* * *} & -1,954^{* * *} \\ (0,413) & (0,316) & (0,368) & (0,363) & (0,263) & (0,263)\end{array}$

Não Paramétrico

$\begin{array}{cccccc}-1,215^{* * *} & -1,146^{* * *} & -1,294^{* * *} & -1,297^{* * *} & -0,977^{* * *} & -0,921^{* * *} \\ (0,471) & (0,281) & (0,410) & (0,410) & (0,311) & (0,311) \\ -1,211 * * * & -1,136^{* * *} & -1,138^{* * *} & -1,158^{* * *} & -0,948^{* * *} & -1,020^{* * *} \\ (0,493) & (0,384) & (0,444) & (0,444) & (0,334) & (0,333) \\ -2,439 * * * & -2,369^{* * *} & -2,377^{* * *} & -2,389 * * * & -1,926^{* * *} & -1,967 * * \\ (0,411) & (0,314) & (0,363) & (0,364) & (0,262) & (0,262)\end{array}$

\begin{tabular}{lccccccc}
$\begin{array}{l}\text { Especifica- } \\
\text { ção }\end{array}$ & Linear & Linear & Linear & $\begin{array}{c}\text { Quadrá- } \\
\text { tico }\end{array}$ & $\begin{array}{c}\text { Quadrá- } \\
\text { tico }\end{array}$ & $\begin{array}{c}\text { Quadrá- } \\
\text { tico }\end{array}$ & $\begin{array}{c}\text { Quadrá- } \\
\text { tico }\end{array}$ \\
Bandwidth & CCT & IK & CV & CCT & IK & CV & CCT \\
N. Obs. & 1.718 & 5.019 & 2.702 & 2.703 & 33.451 & 38.137 & 389 \\
\hline
\end{tabular}

Fonte: Elaboração própria.

Nota: Var. Dependente Mortalidade Infantil. Todas especificações usam Kernel Triangular. O PAR estima a descontinuidade de municípios logo acima de 100.000 habitantes. CCT refere-se a seleção de bandwidth de Calonico, Cattaneo e Titiunik (2014); IK é de Imbens e Kalyanaraman (2012); CV é o método Cross-validation de Ludwig e Miller (2007). A coluna 7 é um teste placebo de um ano antes do inicio do PAR. Erro-Padrão robusto em parênteses. ** *p<0,01; ** $\mathrm{p}<0,05 ;$ * $\mathrm{p}>0,1$.

No entanto, sabemos que o efeito da redução das mortes muitas vezes está relacionado com o tempo que a ação do mecanismo leva para agir. Como resposta, verificamos que acontece a redução das mortes nos três anos subsequentes ao início do PAR, logo esses resultados são estatisticamente significantes. A magnitude 
de redução das mortes nos três anos foi de $11,3 \%, 11,8 \%$ e de $26,1 \%$, respectivamente, nos três anos após o início do PAR no município.

Para atingirmos resultados mais precisos, inserimos controles nas regressões e apresentamos os resultados na Tabela 3. Percebe-se que os resultados não fogem do efeito encontrado na Tabela 2, que apresenta uma redução das mortes infantis, mas o que temos agora é uma maior precisão nos valores encontrados, lembrando também ser estatisticamente significante. Assim, com a inclusão dos controles, a redução das mortes é de $11 \%$. Para os demais anos após a entrada do PAR, há as seguintes reduções: de $13,1 \%$ no primeiro ano; de 12,8\%, para o segundo ano; e de $25,8 \%$ para o terceiro ano.

Tabela 3 - RD Estimativas do Impacto do PAR sobre a Mortalidade Infantil - Com Variáveis de Controle

\begin{tabular}{llllllll}
\hline Variável & (1) & (2) & (3) & (4) & (5) & (6) & (7) \\
\hline
\end{tabular}

Painel A: Efeito do PAR

Paramétrico

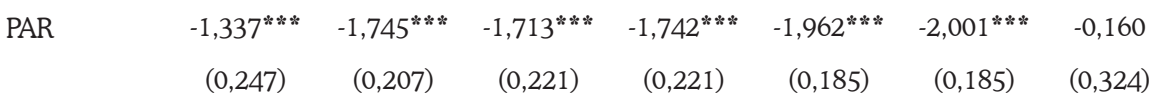

Não Paramétrico

\begin{tabular}{|c|c|c|c|c|c|}
\hline$-1,321 * * *$ & $-1,755^{* * *}$ & $-1,728^{* * *}$ & $-1,808^{* * *}$ & $-1,954^{* * *}$ & $-1,983^{* * * *}$ \\
\hline$(0,247)$ & $(0,213)$ & $(0,221)$ & $(0,207)$ & $(0,186)$ & $(0,136)$ \\
\hline
\end{tabular}

Painel B: Efeito do PAR - Anos Posteriores

Paramétrico

$\begin{array}{cccccc}-1,417^{* * *} & -1,088^{* * *} & -1,303^{* * *} & -1,321 * * * & -0,727 * & -0,657 * * * \\ (0,452) & (0,386) & (0,417) & (0,417) & (0,380) & (0,376) \\ -1,306^{* * *} & -1,179 * * * & -1,144^{* * *} & -1,174 * * * & -0,692 * * * & -0,759 * \\ (0,509) & (0,421) & (0,481) & (0,482) & (0,406) & (0,402) \\ -2,460 * * * & -2,356^{* * *} & -2,334 * * * & -2,379 * * * & -1,688^{* * *} & -1,762 * * * \\ (0,401) & (0,331) & (0,378) & (0,378) & (0,315) & (0,314)\end{array}$

Não Paramétrico

continua... 
conclusão.

\begin{tabular}{lccccccc}
\hline Variável & $\mathbf{( 1 )}$ & $\mathbf{( 2 )}$ & $\mathbf{( 3 )}$ & $\mathbf{( 4 )}$ & $\mathbf{( 5 )}$ & $\mathbf{( 6 )}$ & $\mathbf{( 7 )}$ \\
\hline & $-1,417^{* * *}$ & $-1,034^{* * *}$ & $-1,297^{* * *}$ & $-1,311^{* * *}$ & $-0,732^{* * *}$ & $-0,653^{* * * *}$ & - \\
& $(0,452)$ & $(0,356)$ & $(0,412)$ & $(0,413)$ & $(0,379)$ & $(0,375)$ & \\
\hline Não Paramétrico & & & & & & & \\
& $-1,277^{* * *}$ & $-1,173^{* * *}$ & $-1,128^{* * *}$ & $-1,164^{* * *}$ & $-0,688^{*}$ & $-0,751^{* *}$ & - \\
& $(0,506)$ & $(0,421)$ & $(0,482)$ & $(0,480)$ & $(0,407)$ & $(0,403)$ & \\
& $-2,418^{* * *}$ & $-2,358^{* * *}$ & $-2,328^{* * *}$ & $-2,369^{* * *}$ & $-1,700^{* * *}$ & $-1,764^{* * *}$ & - \\
& $(0,400)$ & $(0,331)$ & $(0,378)$ & $(0,377)$ & $(0,316)$ & $(0,403)$ & \\
Especifica- & & & & & & & \\
ção & Linear & Linear & Linear & Quadrá- & Quadrá- & Quadrá- & Quadrá- \\
Bandwidth & CCT & IK & CV & CCT & IK & CV & CCT \\
N. Obs. & 1.718 & 5.019 & 2.702 & 2.703 & 33.451 & 38.137 & 389 \\
\hline
\end{tabular}

Fonte: Elaboração própria.

Nota: Var. Dependente Mortalidade Infantil. Todas especificações usam Kernel Triangular. O PAR estima a descontinuidade de municípios logo acima de 100.000 habitantes. CCT refere-se a seleção de bandwidth de Calonico, Cattaneo e Titiunik (2014); IK é de Imbens e Kalyanaraman (2012); CV é o método Cross-validation de Ludwig e Miller (2007). A coluna 7 é um teste placebo de um ano antes do inicio do PAR. Erro-Padrão robusto em parênteses. $* *$ p $<0,01 ; *{ }^{*} \mathrm{p}<0,05 ;{ }^{*} \mathrm{p}>0,1$.

A possibilidade de que tendências temporais ou outros programas e ações possam interferir nos resultados de redução das mortes, fazendo com que os efeitos encontrados não advenham do PAR, foram eliminados quando observamos os resultados da coluna sete das Tabelas 2 e 3 . Os resultados dessas colunas não demonstraram qualquer significância estatística, propondo que antes do PAR não havia qualquer efeito influenciando nas mortes infantis.

Os resultados apresentados até o momento demonstram que o PAR consegue reduzir as mortes infantis não somente no ano de seu início, mas também nos anos subsequentes. Porém, ainda podem restar dúvidas quanto à especificação do modelo, como também se existem alguns outros fatores que venham a influenciar sobre a redução da mortalidade infantil. Para confirmar e dirimir essas dúvidas, foram aplicados testes de robustez, apresentados na próxima seção.

\subsection{Testes de Robustez}

Para comprovar a precisão da especificação e garantir os resultados atingidos neste trabalho, aplicamos alguns testes de robustez. A Tabela 4 apresenta um teste no qual alteramos o valor junto ao ponto de corte: onde antes era de 100.000 habitantes, 
conforme critérios do programa, alteramos, inicialmente, para 90.000 e, depois, para 110.000 habitantes, considerando cutoffs falsos. Assim, como esperado, os resultados não foram significativos estatisticamente para esses pontos de cortes falsos.

Tabela 4 - Teste de Robustez do Impacto do PAR sobre a Mortalidade Infantil Alteração de Cutoff.

Variável

(1)

(2)

(3)

(4)

Painel A: Efeito do PAR

Paramétrico

PAR

$$
1,025
$$

$-0,648$

1,175

$-0,261$

$(1,246)$

$(3,628)$

$(1,230)$

$(3,642)$

Não Paramétrico

PAR

$$
-0,920
$$

$-0,523$

$-0,914$

$-0,245$

$(0,635)$

$(3,638)$

$(0,655)$

$(3,669)$

Painel B: Efeito do PAR - Anos Posteriores

Paramétrico

$\begin{array}{cccc}0,186 & -1,602 & 0,345 & -1,599 \\ (1,067) & (2,710) & (1,056) & (2,735) \\ 1,014 & -1,179 & 1,179 & -1,158 \\ (1,081) & (4,145) & (1,145) & (4,095) \\ -0,677 & -2,215 & -0,650 & -2,103 \\ (0,878) & (2,110) & (0,896) & (2,057)\end{array}$

Não Paramétrico

\begin{tabular}{cccc}
0,404 & $-1,607$ & 0,390 & $-1,605$ \\
$(0,845)$ & $(2,736)$ & $(0,870)$ & $(2,761)$ \\
$-0,023$ & $-1,114$ & $-0,028$ & $-1,123$ \\
$(0,652)$ & $(3,916)$ & $(0,676)$ & $(3,974)$ \\
\hline
\end{tabular}


conclusão.

\begin{tabular}{lcccc}
\hline Variável & $\mathbf{( 1 )}$ & $\mathbf{( 2 )}$ & $\mathbf{( 3 )}$ & $\mathbf{( 4 )}$ \\
\hline Não Paramétrico & & & & \\
& $-0,635$ & $-2,227$ & $-0,625$ & $-2,124$ \\
& $(0,611)$ & $(2,089)$ & $(0,628)$ & $(2,059)$ \\
& & & & \\
Controles & Sim & Sim & Sim & Sim \\
Especificação & Linear & Linear & Quadrático & Quadrático \\
Bandwidth & 10.000 & 10.000 & 10.000 & 10.000 \\
Cutoff & 90.000 & 110.000 & 90.000 & 110.000 \\
N. Obs. & 74 & 74 & 74 & 74 \\
\hline
\end{tabular}

Fonte: Elaboração própria.

Nota: Var. Dependente Mortalidade Infantil. Todas especificações usam Kernel Triangular. ErroPadrão robusto em parênteses. ${ }^{* * *} \mathrm{p}<0,01 ;{ }^{* *} \mathrm{p}<0,05 ;{ }^{*} \mathrm{p}>0,1$.

Um segundo teste de robustez alterou a variável dependente para uma causa de morte não relacionada com o PAR a fim de testar mortes por diabetes, neoplasias e abortos. Como resultados, podemos observar, na Tabela 5, que nenhuma coluna apresentou significância estatística e garante que nossa especificação está bem estruturada.

Tabela 5 - Teste de Robustez do Impacto do PAR sobre a Mortalidade Infantil Causa de Morte Placebo.

\begin{tabular}{|c|c|c|c|c|c|c|}
\hline Variável & $\begin{array}{c}\text { Diabe- } \\
\text { tes }\end{array}$ & Neoplasias & Aborto & Diabetes & $\begin{array}{l}\text { Neopla- } \\
\text { sias }\end{array}$ & Aborto \\
\hline
\end{tabular}

Paramétrico

\begin{tabular}{|c|c|c|c|c|c|c|}
\hline PAR & $-0,059$ & $-0,446$ & 17,754 & 0,006 & 0,088 & 3,390 \\
\hline & $(0,107)$ & $(0,400)$ & $(13,754)$ & $(0,093)$ & $(0,524)$ & $(11,770)$ \\
\hline
\end{tabular}

Não Paramétrico

\begin{tabular}{|c|c|c|c|c|c|c|}
\hline PAR & $-0,109$ & $-0,443$ & 17,216 & $-0,003$ & 0,041 & 1,791 \\
\hline & $(0,112)$ & $(0,667)$ & $(13,719)$ & $(0,092)$ & $(0,526)$ & $(11,730)$ \\
\hline
\end{tabular}

Paramétrico

\begin{tabular}{cccccc}
$-0,056$ & 0,161 & $-12,716$ & $-0,089$ & $-0,684$ & $-15,078$ \\
$(0,141)$ & $(0,658)$ & $(18,754)$ & $(0,121)$ & $(0,687)$ & $(16,396)$ \\
\hline
\end{tabular}


conclusão.

\begin{tabular}{|c|c|c|c|c|c|c|}
\hline Variável & $\begin{array}{c}\text { Diabe- } \\
\text { tes }\end{array}$ & Neoplasias & Aborto & Diabetes & $\begin{array}{l}\text { Neopla- } \\
\text { sias }\end{array}$ & Aborto \\
\hline \multicolumn{7}{|l|}{ Paramétrico } \\
\hline & $-0,083$ & 0,736 & $-14,065$ & $-0,057$ & $-0,215$ & $-19,898$ \\
\hline & $(0,139)$ & $(0,778)$ & $(20,695)$ & $(0,119)$ & $(0,771)$ & $(18,110)$ \\
\hline & $-0,089$ & 0,257 & $-17,939$ & $-0,027$ & $-0,542$ & $-19,486$ \\
\hline & $(0,125)$ & $(0,637)$ & $(16,504)$ & $(0,107)$ & $(0,642)$ & $(14,572)$ \\
\hline \multicolumn{7}{|c|}{ Não Paramétrico } \\
\hline & 0,054 & 0,151 & $-12,845$ & $-0,097$ & $-0,696$ & $-15,271$ \\
\hline & $(0,140)$ & $(0,657)$ & $(13,670)$ & $(0,121)$ & $(0,686)$ & $(16,201)$ \\
\hline & $-0,811$ & 0,734 & $-14,135$ & $-0,058$ & $-0,182$ & $-18,641$ \\
\hline & $(0,138)$ & $(0,774)$ & $(20,594)$ & $(0,092)$ & $(0,769)$ & $(17,939)$ \\
\hline & $-0,085$ & 0,241 & $-17,971$ & $-0,035$ & $-0,532$ & $-18,876$ \\
\hline & $(0,125)$ & $(0,632)$ & $(16,468)$ & $(0,107)$ & $(0,643)$ & $(14,482)$ \\
\hline $\begin{array}{l}\text { Especifica- } \\
\text { ção }\end{array}$ & Linear & Linear & Linear & Quadrático & Quadrático & Quadrático \\
\hline Bandwidth & CCT & CCT & CCT & $\mathrm{CCT}$ & $\mathrm{CCT}$ & CCT \\
\hline Controles & Sim & Sim & $\operatorname{Sim}$ & Sim & Sim & Sim \\
\hline N. Obs. & 1.590 & 1.590 & 1.590 & 2.489 & 2.489 & 2.489 \\
\hline
\end{tabular}

Fonte: Elaboração própria.

Nota: Var. Dependente placebo nas colunas. Todas especificações usam Kernel Triangular. O PAR estima a descontinuidade de municípios logo acima de 100.000 habitantes. CCT referese a seleção de bandwidth de Calonico, Cattaneo e Titiunik (2014). Erro-Padrão robusto em parênteses. ${ }^{* * *} \mathrm{p}<0,01 ;{ }^{* *} \mathrm{p}<0,05 ;{ }^{*} \mathrm{p}>0,1$.

Aplicamos mais um teste de robustez no qual alteramos a função kernel da regressão, passando de triangular para Epanechnikov e cujos resultados se encontram na Tabela 6. Posteriormente, também alteramos para a função kernel uniforme, cujos resultados constam na Tabela 7. Ambos os testes devem ter resultados significativos estatisticamente e, assim, confirmam os resultados antes levantados nas Tabelas 2 e 3. 
Tabela 6 - Teste de Robustez do Impacto do PAR sobre a Mortalidade Infantil Função de Kernel Epanechnikov.

\begin{tabular}{lllllll}
\hline Variável & (1) & (2) & (3) & (4) & (5) & (6) \\
\hline \hline
\end{tabular}

Paramétrico

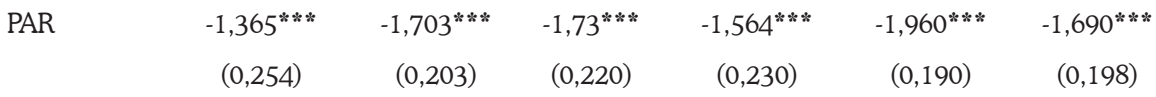

Não Paramétrico

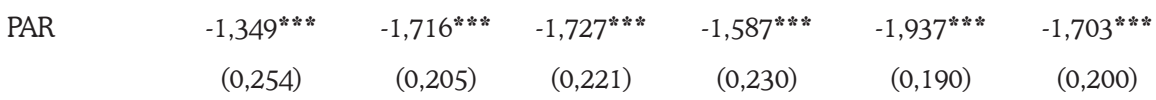

Não Paramétrico

Paramétrico

$\begin{array}{cccccc}-1,493^{* * *} & -1,118^{* * *} & -1,303^{* * *} & -1,213^{* * *} & -0,769^{* *} & -1,043^{* * *} \\ (0,467) & (0,395) & (0,417) & (0,427) & (0,398) & (0,395) \\ -1,119^{* * *} & -1,197^{* * *} & -1,144^{* * *} & -1,079^{* *} & -0,724^{*} & -1,223^{* * *} \\ (0,516) & (0,385) & (0,481) & (0,488) & (0,417) & (0,444) \\ -2,528^{* * *} & -2,333^{* * *} & -2,334^{* * *} & -2,269^{* * *} & -1,755^{* * *} & -2,346^{* * *} \\ (0,411) & (0,316) & (0,378) & (0,390) & (0,329) & (0,343)\end{array}$

Não Paramétrico

$\begin{array}{cccccc}-1,491^{* * *} & -1,131^{* * *} & -1,311^{* * *} & -1,249^{* * *} & -0,779^{* * *} & -1,054^{* * *} \\ (0,466) & (0,395) & (0,351) & (0,427) & (0,397) & (0,395) \\ -1,119^{* *} & -1,212^{* * *} & -1,158^{* *} & -1,098^{* *} & -0,738^{* * *} & -1,233^{* * *} \\ (0,515) & (0,430) & (0,481) & (0,486) & (0,418) & (0,444) \\ -2,539^{* * *} & -2,342^{* * *} & -2,356^{* * *} & -2,314^{* * *} & -1,779 * * * & -2,359^{* * *} \\ (0,409) & (0,341) & (0,378) & (0,389) & (0,329) & (0,343)\end{array}$

\begin{tabular}{lcccccc}
$\begin{array}{l}\text { Especifica- } \\
\text { ção }\end{array}$ & Linear & Linear & Linear & Quadrático & Quadrático & Quadrático \\
Bandwidth & CCT & IK & CV & CCT & IK & CV \\
\hline
\end{tabular}


conclusão.

\begin{tabular}{lcccccc}
\hline Controles & Sim & Sim & Sim & Sim & Sim & Sim \\
N. Obs. & 1.414 & 3.783 & 2.488 & 2.245 & 13.888 & 3.443 \\
\hline \hline
\end{tabular}

Fonte: Elaboração própria.

Nota: Var. Dependente Mortalidade Infantil. Todas especificações usam Kernel Epanechnikov. O PAR estima a descontinuidade de municípios logo acima de 100.000 habitantes. CCT refere-se a seleção de bandwidth de Calonico, Cattaneo e Titiunik (2014); IK é de Imbens e Kalyanaraman (2012); CV é o método Cross-validation de Ludwig e Miller (2007). Erro-Padrão robusto em parênteses. ${ }^{* * *} \mathrm{p}<0,01 ;{ }^{* *} \mathrm{p}<0,05 ;{ }^{*} \mathrm{p}>0,1$.

Observamos, tanto na Tabela 6 quanto na Tabela 7, que os resultados apresentam redução do número de mortes, sendo estatisticamente garantidos pela significância. Durante os anos após o início do programa, a tendência de redução permanece e também é significativa estatisticamente. Isso garante, mais uma vez, que a especificação do modelo realmente demonstra o fato de o PAR estar reduzindo as mortes infantis.

Tabela 7 - Teste de Robustez do Impacto do PAR sobre a Mortalidade Infantil Função de Kernel Uniforme.

\begin{tabular}{lllllll}
\hline Variável & (1) & (2) & (3) & (4) & (5) & (6) \\
\hline
\end{tabular}

Paramétrico

\begin{tabular}{|c|c|c|c|c|c|c|}
\hline PAR & $-1,243 * * *$ & $-1,684 * * *$ & $-1,482 * * *$ & $-1,448 * * *$ & $-1,800 * * *$ & $-1,721 * * *$ \\
\hline & $(0,264)$ & $(0,215)$ & $(0,221)$ & $(0,231)$ & $(0,197)$ & $(0,209)$ \\
\hline
\end{tabular}

Não Paramétrico

\begin{tabular}{|c|c|c|c|c|c|c|}
\hline \multirow[t]{2}{*}{ PAR } & $-1,180 * * *$ & $-1,695 * * *$ & $-1,476 * * *$ & $-1,470 * * *$ & $-1,736 * * *$ & $-1,736 * * *$ \\
\hline & $(0,264)$ & $(0,216)$ & $(0,219)$ & $(0,234)$ & $(0,191)$ & $(0,212)$ \\
\hline
\end{tabular}

Paramétrico

$\begin{array}{cccccc}-1,542 * * * & -1,111 * * * & -1,179 * * * & -1,352 * * * & -0,931 * * & -1,067 * * * \\ (0,467) & (0,415) & (0,429) & (0,438) & (0,393) & (0,410) \\ -1,125 * * & -1,088 * * & -1,057 * * & -1,102 * * & -1,180 * * * & -1,086 * * \\ (0,556) & (0,469) & (0,489) & (0,517) & (0,409) & (0,460) \\ -2,577 * * * & -2,313 * * * & -2,227 * * * & -2,392 * * * & -2,170 * * * & -2,333 * * * \\ (0,452) & (0,369) & (0,389) & (0,391) & (0,340) & (0,359)\end{array}$


conclusão.

\begin{tabular}{lcccccc}
\hline Variável & $\mathbf{( 1 )}$ & $\mathbf{( 2 )}$ & $\mathbf{( 3 )}$ & $\mathbf{( 4 )}$ & $\mathbf{( 5 )}$ & $\mathbf{( 6 )}$ \\
\hline Não Paramétrico & & & & & & \\
& $-1,551^{* * *}$ & $-1,115^{* * *}$ & $-1,177^{* * *}$ & $-1,376^{* * *}$ & $-0,911^{* *}$ & $-1,081^{* * *}$ \\
& $(0,491)$ & $(0,415)$ & $(0,429)$ & $(0,438)$ & $(0,393)$ & $(0,409)$ \\
& $-1,137^{* *}$ & $-1,095^{* *}$ & $-1,056^{* *}$ & $-1,117^{* *}$ & $-1,170^{* * *}$ & $-1,096^{* * *}$ \\
& $(0,555)$ & $(0,469)$ & $(0,489)$ & $(0,517)$ & $(0,410)$ & $(0,459)$ \\
& $-2,563^{* * *}$ & $-2,330^{* * *}$ & $-2,225^{* * *}$ & $-2,444^{* * *}$ & $-2,151^{* * *}$ & $-2,348^{* * *}$ \\
& $(0,452)$ & $(0,370)$ & $(0,389)$ & $(0,393)$ & $(0,340)$ & $(0,358)$ \\
& & & & & & \\
\hline Especificação & Linear & Linear & Linear & Quadrático & Quadrático & Quadrático \\
Bandwidth & $\mathrm{CCT}$ & $\mathrm{IK}$ & $\mathrm{CV}$ & $\mathrm{CCT}$ & $\mathrm{IK}$ & $\mathrm{CV}$ \\
Controles & Sim & Sim & Sim & Sim & Sim & Sim \\
N. Obs. & 1.058 & 2.688 & 2.162 & 1.949 & 8.389 & 2.887 \\
\hline \hline
\end{tabular}

Fonte: Elaboração própria.

Nota: Var. Dependente Mortalidade Infantil. Todas especificações usam Kernel Uniforme. O PAR estima a descontinuidade de municípios logo acima de 100.000 habitantes. CCT refere-se a seleção de bandwidth de Calonico, Cattaneo e Titiunik (2014); IK é de Imbens e Kalyanaraman (2012); CV é o método Cross-validation de Ludwig e Miller (2007). Erro-Padrão robusto em parênteses. *** $\mathrm{p}<0,01 ;{ }^{* *} \mathrm{p}<0,05 ;{ }^{*} \mathrm{p}>0,1$.

Em resumo, podemos observar que a partir do momento em que os municípios conseguem implantar as ações do PAR, os problemas ligados à mortalidade infantil são minimizados, ou seja, o programa consegue reduzir as mortes das crianças menores de um ano de idade de forma duradoura. É importante salientar que, mesmo não tendo esse objetivo como uma de suas metas, o PAR, consegue contribuir de forma efetiva na redução de um problema social de extrema importância.

\section{Considerações Finais}

O presente trabalho analisou o impacto do PAR sobre a mortalidade infantil. A teoria e os dados estatísticos constatam que as pessoas que moram em condições precárias ou em submoradias apresentam pior qualidade de vida e maiores chances de mortalidade adulta e infantil.

O governo brasileiro, preocupado com o déficit habitacional e os efeitos que este gera nas vidas das pessoas que moram em condições precárias, criou o PAR com o objetivo de proporcionar uma melhor condição de vida para essas pessoas. Com base nesse objetivo, deduzimos que melhores condições de habitação podem provocar melhora no bem-estar e na qualidade de vida, diminuindo as mortes infantis.

Com a preocupação de se encontrar resultados consistentes para o efeito do PAR sobre a mortalidade infantil, a estratégia empírica utilizada foi o desenho 
de regressão descontínua. A utilização desse método é possível, pois o programa apresenta um ponto de corte, onde os municípios com mais de 100.000 habitantes são elegíveis a participarem do PAR, com isso temos um grupo de tratados e de controles com características semelhantes, tornando as estimações robustas a possíveis problemas na regressão. Também foram acrescentadas variáveis de controles com o intuito de se aumentar a precisão dos estimadores.

Assim, os resultados das regressões apontaram que o PAR consegue reduzir as mortes infantis em $11 \%$, e o efeito dessa redução permanece nos anos posteriores ao ano de início do programa. Ressalta-se que esses efeitos não proveem de possíveis causas temporais ou de outros programas, pois verificamos que, antes da implantação do PAR, os resultados não apresentaram significância estatística. Para fortalecer nossos resultados, além do teste de anos anteriores, aplicamos outros testes de robustez. Checamos pontos de cortes falsos, cujo resultado obtido não identificou qualquer significância estatística tanto no corte a 90.000, quanto no corte a 110.000 habitantes. Isso demonstra que o programa tem efeito somente com o ponto de corte estipulado no PAR e que não há outros possíveis efeitos ocorrendo em pontos de cortes diferentes. Outro teste realizado verificou se outros tipos de doenças que não têm relação com questões habitacionais e com o PAR como mortes por diabetes, por neoplasias e abortos - poderiam causar alterações. Os resultados apontaram estimativas sem significância estatística, demonstrando que o modelo está bem especificado. Por último, os resultados foram estimados utilizando-se as funções kernel Epanechnikov e Uniforme, e mesmo com a mudança de função, os resultados foram significativos como esperado.

Por fim, acreditamos que o grande resultado deste trabalho foi verificar que algumas políticas criadas para um determinado fim podem ter um resultado bem diferente daquele que se imagina e, assim, por impactar em outras áreas, pode contribuir para o desenvolvimento de setores e áreas que menos se espera.

\section{Referências}

ACEVEDO-GARCIA, D. et all. Does housing mobility policy improve health? Housing Policy Debate, v. 15, n. 1, p. 49-98, 2004.

ALLEN, C. On the "Physiological Dope" problematic in housing and illness rese-arch: towards a critical realism of home and health. Housing, Theory and Society, v. 17, n. 2, p. 49-67, 2000.

ANGRIST, J. D.; PISCHKE, J. S. Mostly harmless econometrics: an empiricist's companion. Princeton: Princeton University Press, 2008.

BOUILLON, C. P. et al. Un espacio para el desarrollo: los mercados de vivienda en América Latina y el Caribe. Washington: Banco Interamericano de Desarrollo, 2012. 
BRASIL. Ministério das Cidades. Programa arrendamento residencial. Brasília: Esplanada dos Ministérios, 2011. Disponível em: https://www.mdr.gov.br/habitacao/progrmas-e-acoessnh/67-snh-secretaria-nacional/programas-e-acoes/97-programa-de-arrendamento-residencial-par\#portal-siteactions. Acesso em:10 nov. 2014.

BRITO, J. N. S. Retroalimentação do processo de desenvolvimento de empreendimentos de habitação de interesse social a partir de reclamações de usuários: estudo no Programa de Arrendamento Residencial. (Mestrado em Engenharia) - Escola de Engenharia, Universidade Federal do Rio Grande do Sul, Porto Alegre, 2009. Disponível em: http://hdl.handle.net/10183/21267. Acesso em: 9 jun. 2020.

BROOKS-GUNN, J; DUNCAN, G. J.; KLABANOV, P. K.; SEALAND, N. Do neighborhoods influence child and adolescent development? American Journal of Sociology, v. 99, n. 2, p. 353395, 1993.

CAIXA ECONÔMICA FEDERAL. Programa de Arrendamento Residencial. Brasília: CAIXA. Disponível em: www.caixa.gov.br. Acesso em: nov. 2014.

CALONICO, Sebastian; CATTANEO, Matias D.; TITIUNIK, Rocio. Robust nonparametric confidence intervals for regression-discontinuity designs. Econometrica, v. 82, n. 6, p. 22952326, 2014.

CURTIS, L. J.; DOOLEY, M. D.; PHIPPS, S. A. Child well-being and neighbourhood quality: evidence from the Canadian National Longitudinal Survey of Children and Youth. Social Science 8 Medicine, v. 58, n. 10, p. 1917-1927, 2004.

DATASUS. [2013]. Disponível em: http://www2.datasus.gov.br/DATASUS/index. php?area $=0102$. Acesso em: out. 2013.

DUNN, J. R. Housing and health inequalities: review and prospects for research. Housing Studies, v. 15, n. 3, p. 341-366, 2000.

ELLEN, I. G.; MIJANOVICH, T.; DILLMAN, K. Neighborhood effects on health: exploring the links and assessing the evidence. Journal of Urban Affairs, v. 23, n. 34, p. 391-408, 2001.

ELLEN, I. G.; TURNER, M. A. Does neighborhood matter? Assessing recent evidence. Housing Policy Debate, v. 8, n. 4, p. 833-866, 1997.

FUJIWARA, T. Voting technology, political responsiveness, and infant health: evidence from Brazil. Econometrica, v. 83, n. 2, p. 423-464, 2015.

GELMAN, A.; IMBENS, G. Why high-order polynomials should not be used in regression discontinuity designs. NBER Working Paper, n. 20405, 2014.

HENLEY, A. Job creation by the self-employed: the roles of entrepreneurial and financial capital. Small Business Economics, v. 25, n. 2, p. 175-196, 2005.

IMBENS, G. W.; KALYANARAMAN, K. Optimal bandwidth choice for the regression discontinuity estimator. Review of Economic Studies, v. 79, n. 3, 2012. 
IMBENS, G. W.; LEMIEUX, T. Regression discontinuity designs: a guide to practice. Journal of Econometrics, v. 142, n. 2, p. 615-635, 2008.

IBGE. Sistema IBGE de Recuperação Automática - SIDRA. Disponível em: <http:www.sidra. ibge.gov.br>. Acesso em: nov. 2013.

JACOB, B. A.; LUDWIG, J.; MILLER, D. L. The effects of housing and neighborhood conditions on child mortality. Journal of Health Economics, v. 32, n. 1, p. 195-206, 2013.

KAWACHI, I.; BERKMAN, L. F. (ed.). Neighborhoods and health. Oxford: Oxford University Press, 2003.

$\mathrm{KUH}, \mathrm{D}$. et al. Mortality in adults aged 26-54 years related to socioeconomic conditions in childhood and adulthood: post war birth cohort study. The BMJ, v. 325, n. 7372, p. 10761080, 2002.

LEVENTHAL, T.; BROOKS-GUNN, J. The neighborhoods they live in: the effects of neighborhood residence on child and adolescent outcomes. Psychological Bulletin, v. 126, n. 2, p. 309, 2000.

LUDWIG, J.; MILLER, D. L. Does head start improve children's life chances? Evidence from a regression discontinuity design. NBER Working Paper, n. 11702, 2007

MACINTYRE, S.; ELLAWAY, A. Ecological approaches: rediscovering the role of the physical and social environment. In: BERKMAN, L. F.; KAWACHI, I. Social epidemiology. New York: Oxford University Press, 2000, p. 332-348.

MCCRARY, J. Manipulation of the running variable in the regression discontinuity design: a density test. Journal of Econometrics, v. 142, n. 2, p.698-714, 2008.

MONASTERIO, L. O FPM e a estranha distribuição da população dos pequenos municípios brasileiros. Texto para Discussão - IPEA, n. 1818, 2013.

ONIBOKUN, P. Housing needs and responses: a planners viewpoint on housing in Nigeria. Ibadan: NISER, 1983, p. 65-82.

PENG, R.; WHEATON, W. C. Effects of restrictive land supply on housing in Hong Kong: an econometric analysis. Joint Center for Housing Studies, v. 5, n. 2, 1994.l

PNUD. Atlas do desenvolvimento humano no Brasil. Disponível em: http://atlasbrasil.org. br/2013/. [2013]. Acesso em: nov. 2013.

POLLAKOWSKI, H. O.; WACHTER, S. M. The effects of land-use constraints on housing prices. Land Economics, v. 66, n. 3, p. 315-324, 1990.

RAMÍREZ, R. Evaluación social de políticas y programas de vivienda: un análisis de la contribución de la vivienda a la reducción de la pobreza urbana. Revista Invi, v. 17, n. 45, 2002.

ROBERT, S.; HOUSE, J. S. SES differentials in health by age and alter-native indicators of SES. Journal of Aging and Health, v. 8, n. 3, p. 359-388, 1996. 
ROCHA, V. A.; BELLUZZO, W. Avaliação do programa de descentralização de gastos públicos no sistema municipal de ensino fundamental de São Paulo. In: ENCONTRO NACIONAL DE ECONOMIA - ANPEC, 38., 2010, Salvador. Anais eletrônicos [...]. Salvador: ANPEC, 2010.

RUBIN, G. R. Exposição do problema habitacional em dois países da América Latina. Cadernos ProArq: Revista de Arquitetura e Urbanismo da PROARQ, n. 20, 2013.

SAKS, R. E. Job creation and housing construction: constraints on metropolitan area employment growth. Journal of Urban Economics, v. 64, n. 1, p. 178-195, 2008.

SALAU, A. T. The environmental context of urban housing-public services and infrastructural facilities in Nigerian urban centers. In: ONIBOKUN, P. Urban housing in Nigeria. Ibadan: NISER, 1990, p. 58-88.

SENGOELGE, M. et al. Housing, income inequality and child injury mortality in Europe: a cross-sectional study. Child: Care, Health and Development, v. 40, n. 2, p. 283-291, 2014.

SMITH, A. C. Spring forward at your own risk: daylight saving time and fatal vehicle crashes. American Economic Journal: Applied Economics, v. 8, n. 2, 2016.

TEIXEIRA, L. P.; DE CARVALHO, F. M. A. A construção civil como instrumento do desenvolvimento da economia brasileira. Revista Paranaense de Desenvolvimento, n. 109, p. 9-26, 2005.

TEKCE, B.; SHORTER, F. C. Determinants of child mortality: a study of squatter settlements in Jordan. Population and Development Review, v. 10, p. 257-280, 1984.

THISTLETHWAITE, D. L.; CAMPBELL, D. T. Regression-discontinuity analysis: an alternative to the ex post facto experiment. Journal of Educational Psychology, v. 51, n. 6, p. 309, 1960.

TROCHIM, W. M. K. Research design for program evaluation: the regression-discontinuity approach. California: SAGE Publications Inc, 1984.

UNCED. Promoting sustainable human settlement development. In: UNCED. Earth summit report: agenda 21. [S. 1.: s. n.], 1992.

WADSWORTH, M. E. J.; MONTGOMERY, S. M.; BARTLEY, M. J. The persisting effect of unemployment on health and social well-being in men early in working life. Social Science Medicine, v. 48, n. 10, p. 1491-1499, 1999.

ZHAO, P.; LÜ, B.; ROO, G. Impact of the jobs-housing balance on urban commuting in Beijing in the transformation era. Journal of Transport Geography, v. 19, n. 1, p. 59-69, 2011.

Recebido em: 10/04/2018.

Aceito em: 06/07/2018.

(cc) BY 